\title{
ON SOME ALMOST KÄHLERIAN SPACES
}

\author{
Dedicated to Professor K. Yano on his 50th birthday.
}

\author{
Yosio Muto
}

(Received July 16,1962)

1. Introduction. Let us consider a Riemannian space $V^{2 n}$ with positive definite metric tensor $G_{B A}{ }^{1)}$ and admitting a Killing vector field $u^{A}$ such that the magnitude of the absolute differential $d \xi^{T} \nabla_{T} u^{A}$ is equal to the magnitude of the infinitesimal vector $d \xi^{A}$. Then we get from $\left(d \xi^{B} \nabla_{B} u^{T}\right)\left(d \xi^{A} \nabla_{A} u^{S}\right) G_{T S}=d \xi^{B} d \xi^{A} G_{B A}$ the equation

$$
\left(\nabla_{B} u^{T}\right)\left(\nabla_{A} u_{T}\right)=G_{B A}
$$

where $u_{A}=u^{S} G_{S A}$.

A Killing vector $u^{A}$ satisfies the equation $\nabla_{B} u_{A}+\nabla_{A} u_{B}=0$, so that, if we put

$$
\nabla_{B} u^{A}=F_{B}^{A},
$$

the equation (1. 1) is equivalent to

$$
F_{B}^{r^{T}} F_{T}^{\cdot A}=-\delta_{B}^{A} .
$$

Thus we obtain an almost Hermitian structure. We shall study in the present paper some local properties of spaces $V^{2 n}$ with almost complex structure $F_{B}^{\cdot A}$ which is derived in such a way from a Killing vector $u^{A}$. Letters such as $F_{B}{ }^{A}$ and $f_{j}^{i}$ will always denote almost complex or complex structures. satisfies

Since we get from (1.2) $\nabla c \nabla_{B} u^{A}=\nabla_{C} F_{B}^{{ }_{B}^{A}}$ and since a Killing vector $u^{A}$

$$
\begin{gathered}
\nabla c \nabla_{B} u^{A}=-K_{S C B}{ }^{A} u^{S}, \\
\nabla_{C} F_{B A}+\nabla_{B} F_{A G}+\nabla_{A} F_{C B}=0,
\end{gathered}
$$

we get

which shows that our space $V^{2 n}$ is an almost Kählerian space.

$V^{2 n}$ being an almost Kählerian space, we get $\nabla^{B} F_{B}^{\cdot A}=0$ and hence

1) Indices $A, B, \cdots$ would run from 1 to $2 n$, but, since we prefer a special coordinate system, we use indices as follows,

$$
\begin{aligned}
& A, B, C, \cdots, S, T, \cdots=0,1, \cdots, 2 n-2, \infty, \\
& \alpha, \beta, \gamma, \cdots, \lambda, \mu, \cdots=1, \cdots, 2 n-2, \infty, \\
& p, q, r, \cdots, x, y, z=0,1, \cdots, 2 n-2, \\
& h, i, j, k, l, m, n=1, \cdots, 2 n-2 .
\end{aligned}
$$


$\nabla^{B} \nabla_{B} u^{A}=0 . \quad$ We then obtain $K_{\dot{S}}{ }^{A} u^{S}=0$ where $K_{\dot{B}}{ }^{A}=K_{B S} G^{S A}$ and $K_{B A}$ is the Ricci tensor.

We also obtain

$$
\begin{aligned}
\underset{u}{\mathfrak{E}} F_{B}^{\cdot A}={\underset{u}{\mathfrak{E}}}_{\vec{A}} \nabla_{B} u^{A} & =\left(\underset{u}{\mathfrak{f}} \nabla_{B}-\nabla_{B} \underset{u}{\mathfrak{E}}\right) u^{A}+\nabla_{B} \underset{u}{\mathfrak{f}} u^{A} \\
& =\left(\underset{u}{\mathfrak{f}}\left\{\begin{array}{c}
A \\
B C
\end{array}\right\}\right) u^{C}=0
\end{aligned}
$$

from which we find that $u^{A}$ is a contravariant almost analytic vector [2].

Thus we obtain the

THEOREM 1.1. If a Riemannian space $V^{2 n}$ admits a Killing vector field $u^{4}$ such that the tensor $\nabla_{B} u^{A}$ determines an almost Hermitian structure in $V^{2 n}$, then the space is an almost Kählerian space and the Ricci tensor is not definite. Moreover, the vector $u^{4}$ is a contravariant almost analytic vector.

2. Killing vector and some special coordinate systems. Let us consider that a Killing vector $\mathbf{u}$ is given in a Riemannian space $V^{2 n}$ and assume that its magnitude $|\mathbf{u}|$ is not a constant. We prove in the following that we can then find a coordinate system $\left(\xi^{A}\right)$ such that the components $u^{A}$ of the given Killing vector in this coordinate system satisfy

$$
u^{A}=\delta_{0}^{A}, \quad u^{T} u_{T}=G_{00}=\xi^{\infty} .
$$

Let $\left(\eta^{A}\right)$ be any coordinate system in which the given Killing vector has the components $v^{0}, v^{1}, \ldots, v^{2 n-2}, v^{\infty}$ and the fundamental tensor has the components $H_{B A}$. Then, if we put $\phi^{\infty}=v^{T} v^{S} H_{T S}$, we get

$$
\frac{\partial \varphi^{\infty}}{\partial \eta^{A}} v^{A}=0
$$

for we have

$$
\begin{aligned}
v^{A} \frac{\partial\left(v^{T} v^{S} H_{T S}\right)}{\partial \eta^{A}} & =2 v^{A}\left(\nabla_{A} v^{T}\right) v^{S} H_{T S} \\
& =2\left(\nabla_{A} v_{T}\right) v^{A} v^{T}=0 .
\end{aligned}
$$

Evidently we can choose $2 n-2$ functions $\varphi^{1}, \phi^{2}, \cdots, \phi^{2 n-2}$ of $\eta^{A}$ such that

$$
\frac{\partial \varphi^{1}}{\partial \eta^{A}} v^{A}=0, \ldots \ldots \ldots, \frac{\partial \phi^{2 n-2}}{\partial \eta^{A}} v^{A}=0
$$

and moreover such that the rank of the matrix 


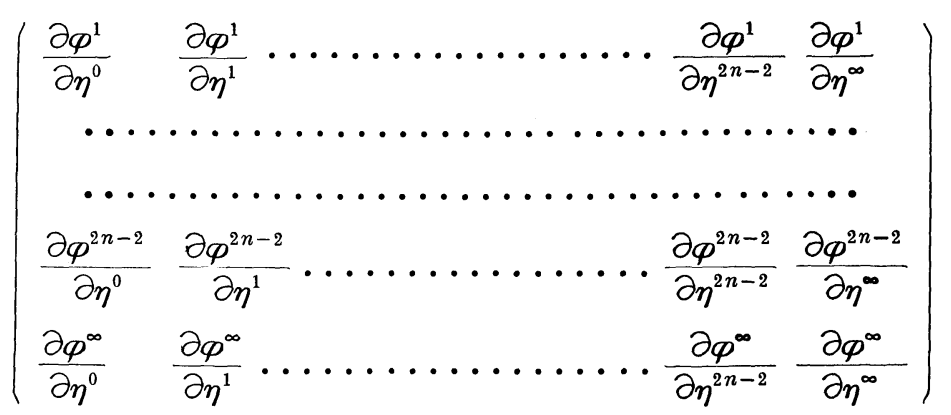

is $2 n-1$. Then, if $\varphi^{0}$ is a solution of the equation

$$
\frac{\partial \varphi^{0}}{\partial \eta^{A}} v^{A}=1
$$

we get

$$
\operatorname{det}\left(\frac{\partial \phi^{A}}{\partial \eta^{B}}\right) \neq 0
$$

so that we can take $\left(\xi^{A}\right)=\left(\phi^{A}\right)$ as a new coordinate system. The components $u^{A}$ of the given Killing vector in this coordinate system satisfy

$$
u^{A}=\frac{\partial \phi^{A}}{\partial \eta^{B}} v^{B}=\delta_{0}^{A}, \quad u^{T} u_{T}=v^{T} v_{T}=\phi^{\infty}=\xi^{\infty}
$$

and we get $G_{00}=G_{B A} u^{B} u^{A}=u^{T} u_{T}=\xi^{\infty}$, hence (2. 1) is satisfied.

We can derive from (2. 1) the following equations ([1], p.209; [3],p. 31 ; [4],p.49) :

$$
\partial_{0} G_{B A}=0
$$

Now consider the equations

$$
\begin{aligned}
G^{0 \infty}+G^{i \infty} \frac{\partial f}{\partial \xi^{i}}+G^{\infty \infty} \frac{\partial f}{\partial \xi^{\infty}} & =0, \\
G^{i \infty} \frac{\partial f^{j}}{\partial \xi^{i}}+G^{\infty \infty} \frac{\partial f^{j}}{\partial \xi^{\infty}} & =0 .
\end{aligned}
$$

As the tensor $G_{B A}$ is positive definite, we have $G^{\infty \infty}>0$. On the other hand, since we have (2. 2), the functions $G^{B .4}$ do not involve the variable $\xi^{0}$. Consequently we can find functions $f, f^{j}$ of $\xi^{1}, \ldots, \xi^{2 n-2}, \xi^{\infty}$ satisfying (2. 3) and

$$
\operatorname{det}\left(\frac{\partial f^{j}}{\partial \xi^{i}}\right) \neq 0
$$

Then 


$$
\left\{\begin{array}{l}
\xi^{0 \prime}=\xi^{0}+f\left(\xi^{1}, \cdots, \xi^{2 n-2}, \xi^{\infty}\right), \\
\xi^{j \prime}=f^{j}\left(\xi^{1}, \cdots, \xi^{2 n-2}, \xi^{\infty}\right), \\
\xi^{\infty \prime}=\xi^{\infty}
\end{array}\right.
$$

is a coordinate transformation such that the components $u^{A^{\prime}}$ of $\mathbf{u}$ and the components $G_{B^{\prime} A^{\prime}}, G^{B^{\prime} A^{\prime}}$ of the fundamental tensor in the new coordinate system $\left(\xi^{A^{\prime}}\right)$ satisfy

$$
\begin{gathered}
u^{A^{\prime}}=\delta_{0^{\prime}}^{A \prime}, \quad \partial_{0^{\prime}} G^{B^{\prime} A^{\prime}}=0, \\
G_{0^{\prime} 0^{\prime}}=\xi^{\infty \prime}, \quad G^{0 \infty \prime}=G_{0^{\prime \infty}}=0, \quad G^{j_{\infty}^{\prime}}=G_{j^{\prime \infty}}=0 .
\end{gathered}
$$

Thus we obtain the

THEOREM 2.1. If a Riemannian space $V^{2 n}$ admits a Killing vector $\mathbf{u}$ whose magnitude $|\mathbf{u}|$ is not a constant in the $V^{2 n}$, then there exists a coordinate system $\left(\xi^{A}\right)$ such that the components $u^{A}$ of $\mathbf{u}$ and the components $G_{B A}$ of the fundamental tensor in this coordinate system satisfy the equations

$$
\begin{array}{ll}
u^{A}=\delta_{0}^{A}, & \partial_{0} G_{B A}=0, \\
G_{00}=\xi^{\infty}, & G_{0 \infty}=G_{i \infty}=0 .
\end{array}
$$

Such a coordinate system will be called a favourable coordinate system in the present paper.

There are many favourable coordinate systems. If $\left(\xi^{A}\right)$ and $\left(\xi^{A \prime}\right)$ are such ones, then we have

$$
\left\{\begin{array}{l}
\xi^{0 \prime}=\xi^{0}+f\left(\xi^{1}, \cdots, \xi^{2 n-2}\right) \\
\xi^{i \prime}=f^{i \prime}\left(\xi^{1}, \cdots, \xi^{2 n-2}\right) \\
\xi^{\infty \prime}=\xi^{\infty}
\end{array}\right.
$$

REMARK 1. If (2.1) is satisfied, we have

$$
\left(\nabla_{B}\left(u^{T} u_{T}\right)\right)\left(\nabla_{A} \varphi\right) G^{B A}=\left(\nabla_{B} \xi^{\infty}\right)\left(\nabla_{A} \varphi\right) G^{B A}=G^{\infty A} \nabla_{A} \varphi
$$

for any scalar field $\varphi$. Hence we can think that (2.3) means that $\operatorname{grad} \varphi$ and $\operatorname{grad}\left(u^{T} u_{T}\right)$ are orthogonal to each other whenever we put $\varphi=\xi^{0}+f$ or $\varphi=f^{j}$.

REMARK 2. As $G^{0 \infty}=G^{i \infty}=0$ and $G_{0 \infty}=G_{i \infty}=0$ are equivalent, we find that the hypersurfaces $\xi^{x}=$ const. are intersected orthogonally by the hypersurfaces $u^{T} u_{T}=$ const. and that the parametric curves $\xi^{x}$ are orthogonal to the parametric curves $\xi^{\infty}$ for every number $x=0,1, \cdots, 2 n-2$ if we take a favourable coordinate system.

3. A Riemannian space $V^{2 n}$ which admits a Killing vector field $u^{A}$ satisfying the equation $\left(\nabla_{B} u^{T}\right)\left(\nabla_{A} u_{T}\right)=G_{B A}$. At first we prove the

THEOREM 3.1. In a Riemannian space $V^{2 n}$ in which $\nabla_{B} u^{A}$ determines 
an almost Kählerian structure ${ }^{2)}$ the Killing vector $u^{A}$ can not have constant length.

PROOF. If we assume $u^{T} u_{T}=$ const., we get $F_{\dot{A}}{ }^{T} u_{T}=0$ from $\left(\nabla_{A} u^{T}\right) u_{T}=0$. But, as we have $\operatorname{det}\left(F_{B}{ }^{A}\right)= \pm 1$, this leads to contradiction.

The following corollary is a direct consequence of Theorems 2.1 and 3.1.

COROLLARY. In a Riemannian space $V^{2 n}$ in which $\nabla_{B} u^{A}$ determines an almost Kählerian structure we can choose a coordinate system $\left(\xi^{0}, \xi^{i}, \xi^{\infty}\right)$ such that the components $u^{A}$ of the Killing vector $\mathbf{u}$ satisfy

$$
u^{A}=\delta_{0}^{A}
$$

and the components $G_{B A}$ of the metric tensor satisfy

$$
G_{00}=\xi^{\infty}, \quad \partial_{0} G_{B A}=0, \quad G_{0 \infty}=G_{i \infty}=0 .
$$

In such a favourable coordinate system we can write (1.1) in the form

$$
[0 B, T][0 A, S] G^{T S}=G_{B A}
$$

or in the form

$$
\left(\partial_{B} G_{0 T}-\partial_{T} G_{0 B}\right)\left(\partial_{A} G_{0 S}-\partial_{S} G_{0 A}\right) G^{T S}=4 G_{B A} .
$$

Taking (2. 2) into account we get from (3.4)

$$
\begin{aligned}
& \left(\partial_{B} G_{00}\right)\left(\partial_{A} G_{00}\right) G^{00}+\left(\partial_{B} G_{00}\right)\left(\partial_{A} G_{0 \alpha}-\partial_{\alpha} G_{0 A}\right) G^{0 \alpha} \\
& +\left(\partial_{B} G_{03}-\partial_{\beta} G_{0 B}\right)\left(\partial_{A} G_{00}\right) G^{0.3} \\
& +\left(\partial_{B} G_{0 \beta}-\partial_{\beta} G_{0 B}\right)\left(\partial_{A} G_{0 \alpha}-\partial_{\alpha} G_{0 A}\right) G^{\beta \alpha}=4 G_{B A}
\end{aligned}
$$

and taking $G_{00}=\xi^{\infty}, G_{0 \infty}=G_{i \infty}=G^{0 \infty}=G^{i \infty}=0$ into account we find that these equations split into the following three sets of equations,

$$
\begin{gathered}
G^{\infty \infty}=4 G_{00}, \\
G^{\infty \infty} \partial_{\infty} G_{0 \lambda}=4 G_{0 \lambda}, \\
\delta_{\mu}^{\infty} \delta_{\lambda}^{\infty} G^{00}+\delta_{\mu}^{\infty}\left(\partial_{\lambda} G_{0 h}-\partial_{h} G_{0 \lambda}\right) G^{0 h}+\delta_{\lambda}^{\infty}\left(\partial_{\mu} G_{0 k}-\partial_{k} G_{0 \mu}\right) G^{0 k} \\
+\left(\partial_{\mu} G_{0 \beta}-\partial_{\beta} G_{0 \mu}\right)\left(\partial_{\lambda} G_{0 \alpha}-\partial_{\alpha} G_{0 \lambda}\right) G^{\beta \alpha}=4 G_{\mu \lambda} .
\end{gathered}
$$

We obtain $G^{\infty \infty}=4 \xi^{\infty}$ from (3. 5) and substituting this into (3. 6) we have

$$
\xi^{\infty} \partial_{\infty} G_{0 \lambda}=G_{0 \lambda},
$$

hence the functions $f_{A}=\left(\xi^{\infty}\right)^{-1} G_{0.4}$ depend only upon the variables $\xi^{1}, \ldots, \xi^{2 n-2}$. Thus we get

$$
G_{0 A}=\xi^{\infty} f_{A}\left(\xi^{1}, \cdots, \xi^{2 n-2}\right)
$$

2) It follows immediately that $u^{A}$ is then a Killing vector. 
and also

$$
G_{\infty \infty}=\frac{1}{4 \xi^{\infty}}, \quad G^{\infty \infty}=4 \xi^{\infty} .
$$

Equations obtained from (3. 7) by putting $\lambda=\mu=\infty$ and also by putting $\lambda=\infty, \mu=j$ are satisfied identically because of (3. 8) and (3.10). But, if we put $\lambda=i, \mu=j$ in (3. 7), we get

$$
\begin{gathered}
\left(\partial_{j} G_{0 k}-\partial_{k} G_{0 j}\right)\left(\partial_{i} G_{0 h}-\partial_{h} G_{0 i}\right) G^{k h} \\
+\left(\partial_{\infty} G_{0 j}\right)\left(\partial_{\infty} G_{0 i}\right) G^{\infty \infty}=4 G_{j i}
\end{gathered}
$$

which we can write also in the following form,

$$
\begin{gathered}
\frac{1}{4}\left(\partial_{j} G_{0 k}-\partial_{k} G_{0 j}\right)\left(\partial_{i} G_{0 h}-\partial_{h} G_{0 i}\right) G^{k h} \\
=G_{j i}-\left(\xi^{\infty}\right)^{-1} G_{0 j} G_{0 i} .
\end{gathered}
$$

Thus we have the

THEOREM 3.2. Let $V^{2 n}$ be a Riemannian space with an almost Kählerian structure determined by $G_{B A}$ and $F_{\dot{B}}{ }^{A}=\nabla_{B} u^{4}$. Then if we take in $V^{2 n}$ a favourable coordinate system $\left(\xi^{A}\right)$, that is, a coordinate system in which (3.1) and (3. 2) hold, we get (3. 8), (3. 10) and (3.11).

THEOREM 3.3. If a Riemannian space $V^{2 n}$ with the fundamental tensor $G_{B A}$ admits a coordinate system $\left(\xi^{A}\right)$ such that

$$
\partial_{0} G_{B A}=0, G_{00}=\xi^{\infty}, G_{0 \infty}=G_{i \infty}=0, G_{\infty \infty}=\left(4 \xi^{\infty}\right)^{-1},
$$

and moreover such that (3. 8) and (3.11) hold, then a vector $\mathbf{u}$ with the components $u^{A}=\delta_{0}^{A}$ is a Killing vector which satisfies (1. 1) and this $V^{2 n}$ becomes an almost Kählerian space by virtue of the tensor $\nabla_{B} u^{4}$.

THEOREM 3.4. A necessary and sufficient condition that, in a Riemannian space $V^{2 n}$ which admits a Killing vector field $\mathbf{u}, \nabla_{B} u^{A}$ can determine an almost Hermitian structure is that the following two conditions (I) and (II) be fulfilled. (I) $|\mathbf{u}|$ is not constant in $V^{2 n}$ and consequently we can take a favourable coordinate system. (II) The metric tensor $G_{B A}$ of $V^{2 n}$ satisfies (3.8), (3.10) and (3.11) in this coordinate system.

4. A family of almost Kählerian spaces $\stackrel{*}{V^{2 n-2}}$ induced by the Killing vector field $u^{A}$ of $V^{2 n}$. Let us define $g_{\mu \lambda}$ by

$$
g_{\mu \lambda}=\frac{G_{\mu \lambda}}{G_{00}}-\frac{G_{0 \mu} G_{0 \lambda}}{\left(G_{00}\right)^{2}} .
$$

As det $\left(g_{\mu \lambda}\right) \neq 0$, we can define $g^{\mu \lambda}$ by $g_{\nu \alpha} g^{\alpha \lambda}=\delta_{\nu}^{\lambda}$. Then we get

$$
g^{\mu \cdot \lambda}=G_{00} G^{\mu \lambda}
$$


and

$$
\begin{aligned}
& G^{00}=\frac{1}{G_{00}}+\frac{g^{3 \alpha} G_{09} G_{0 \alpha}}{\left(G_{00}\right)^{3}}, \\
& G^{0 \lambda}=-\frac{g^{\lambda \alpha} G_{0 \alpha}}{\left(G_{00}\right)^{2}}, \\
& G_{\mu \lambda}=G_{00} g_{\mu \lambda}+\frac{G_{0 \mu} G_{0 \lambda}}{G_{00}} .
\end{aligned}
$$

Using these equations and (3.9) we can write (3.11) in the form

$$
\frac{1}{4}\left(\partial_{j} f_{k}-\partial_{k} f_{j}\right)\left(\partial_{i} f_{h}-\partial_{h} f_{i}\right) g^{k h}=g_{j i} \text {. }
$$

Since we have $g_{i \infty}=0$ because of $G_{0 \infty}=G_{i \infty}=0$, we get $g^{k h} g_{h i}=\delta_{i}^{k} . \quad g_{j i}$ do not involve the variable $\xi^{0}$.

Now consider for each value of $\xi^{\infty}$ a Riemannian space of dimension $2 n-2$ in which any point is denoted by the coordinates $\xi^{1}, \ldots, \xi^{2 n-2}$ and the fundamental tensor by $g_{j i}$. As we have (4.4), this Riemannian space admits an almost Hermitian structure determined by

$$
f_{j i}=\frac{1}{2}\left(\partial_{j} f_{i}-\partial_{i} f_{j}\right) .
$$

We denote this almost Hermitian space by $V^{* 2 n-2}\left(\xi^{\infty}\right)$.

In $V^{2 n}$ let $T$ denote a trajectory of the group of motions induced by the Killing vector $u^{A}$. If a trajectory $T$ passes a point $P$, it will be denoted by $T(P)$. Let the coordinates of a point $P$ be $\xi^{A}$ and the coordinates of a point $P^{\prime}$ be $\xi^{A}+d \xi^{A}$. Then we can define the infinitesimal distance between the two trajectories $T(P)$ and $T\left(P^{\prime}\right)$ by the length of the infinitesimal vector

$$
d \xi^{A}-\frac{u_{S} d \xi^{S}}{u^{T} u_{T}} u^{A}
$$

hence by

$$
\left(G_{B A} d \xi^{B} d \xi^{A}-\frac{u_{B} u_{A}}{u^{T} u_{T}} d \xi^{B} d \xi^{A}\right)^{1 / 2}
$$

The distance thus defined depends only upon the trajectories themselves and does not depend upon the position of the points $P, P^{\prime}$ in the trajectories. This is one of the direct consequences of the definition of group of motions.

Consequently we can derive a Riemannian space of dimension $2 n-1$ by regarding each trajectory as a point. This space is denoted by $V_{B}$. Substituting $u_{A}=G_{B A} u^{B}=G_{0 A}$ into (4.6) we find that the fundamental tensor of $V_{B}$ is given by 


$$
G_{\mu \lambda}-\frac{G_{0 u} G_{0 \lambda}}{G_{00}}
$$

if each point of $V_{B}$ is denoted by the coordinates $\xi^{1}, \ldots, \xi^{2 n-2}, \xi^{\infty}$.

Since $\xi^{\infty}$ is constant along each trajectory on account of $\xi^{\infty}=u^{T} u_{T}, V_{B}$ admits a family of hypersurfaces $\xi^{\infty}=$ const. According to (4. 1) and (4. 7) the fundamental tensor of each hypersurface $\xi^{\infty}=c$ is given by $c g_{j i}$ where $g_{j i}$ is the fundamental tensor of $\stackrel{*}{V^{2 n-2}}(c)$.

As the functions $f_{i}=\left(\xi^{\infty}\right)^{-1} G_{0 i}$ do not involve $\xi^{\infty}$, the skew symmetric tensor (4. 5) does not depend upon $\xi^{\infty}$. But, as $g_{j i}$ depends in general upon $\xi^{\infty}$,

$$
f^{j}=g^{j i} f_{i}
$$

and

$$
f_{j}^{i}=f_{j k} g^{k i}=\frac{1}{2}\left(\stackrel{*}{\nabla} f^{i}-\stackrel{*}{\nabla}^{i} f_{j}\right)
$$

involve $\xi^{\infty}$. In (4. 9) $\stackrel{*}{\nabla}_{j}$ denotes covariant differentiation with respect to the Christoffel symbols $\left\{\begin{array}{l}h \\ j i\end{array}\right\}$ formed from $g_{j i}$.

$\stackrel{*}{V}^{2 n-2}$ is such an almost Hermitian space.

But, since we have

$$
\stackrel{*}{\nabla}_{k} f_{j i}+\stackrel{*}{\nabla}_{j} f_{i k}+\stackrel{*}{\nabla} f_{k j}=0
$$

from (4. 5), $\stackrel{*}{V}^{2 n-2}$ is an almost Kählerian space.

We have deduced in such a way a family of almost Kählerian spaces $\stackrel{*}{V}^{2 n-2}\left(\xi^{\infty}\right)$ from a Riemannian space $V^{2 n}$ which admits a Killing vector $u^{A}$ satisfying the equation (1. 1).

Let us consider conversely a family of $(2 n-2)$-dimensional spaces labelled by a parameter $\xi^{\infty}$, so that each space is denoted by $\stackrel{*}{M}\left(\xi^{\infty}\right)$ and the family by

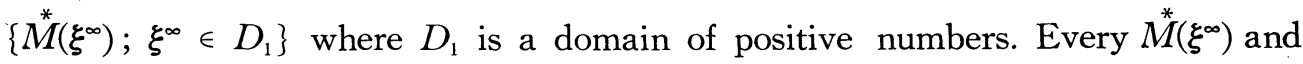
the family are differentiable of class, for example, $C^{\infty}$ and in each $M^{*}\left(\xi^{\infty}\right)$ a fundamental tensor is introduced by the differentiable functions $g_{j i}\left(\xi^{1}, \ldots, \xi^{2 n-2}, \xi^{\infty}\right)$, the coordinates of a point being denoted by $\xi^{1}, \ldots, \xi^{2 n-2}$. We assume that $\stackrel{*}{M}\left(\xi^{\infty}\right)$ becomes an almost Kählerian space by virtue of $g_{j i}$ and the almost complex structure $f_{j}^{i}$ formed from a vector $f_{i}$ by (4. 8) and (4. 9). We assume furthermore that the components $f_{i}$ do not depend upon $\xi^{\infty}$ for some choice of the coordinate system $\left(\xi^{1}, \cdots, \xi^{2 n-2}\right)$ and consider the space $M=\left\{\stackrel{*}{M}\left(\xi^{\infty}\right) \times \xi^{0} \times \xi^{\infty} ; \xi^{0} \in D_{0}\right.$, $\left.\xi^{\infty} \in D_{1}\right\}^{3)}$ where the points are indicated by the coordinates $\xi^{0}, \xi^{1}, \cdots, \xi^{2 n-2}, \xi^{\infty}$.

3) We understand in the right hand side of this equation a union of the point sets $\stackrel{*}{M}\left(\xi^{\infty}\right)$ $\times \xi^{0} \times \xi^{\infty}$ over $\xi^{0} \in D_{0}, \xi^{\infty} \in D_{1}$. In this connection it must be especially emphasized that we are studying only local properties. 
$D_{0}$ is a domain of real numbers. Though each $M^{*}\left(\xi^{\infty}\right)$ is a Riemannian space, we introduce the space $M$ only as a differentiable manifold until we introduce a metric anew.

Now we introduce the fundamental tensor $G_{B A}$ into $M$ by

$$
\begin{aligned}
& G_{00}=\xi^{\infty}, G_{0 \infty}=G_{i \infty}=0, G_{\infty \infty}=\left(4 \xi^{\infty}\right)^{-1}, \\
& G_{0 k}=\xi^{\infty} f_{k}, G_{j i}=\xi^{\infty}\left(g_{j i}+f_{j} f_{i}\right) .
\end{aligned}
$$

Then the space becomes a Riemannian space $V^{2 n}$ which admits evidently a Killing vector $u^{A}=\delta_{0}^{A}$ and this Killing vector satisfies (1. 1). We find immediately that $\stackrel{*}{M}\left(\xi^{\infty}\right)$ can be identified with $V^{* 2 n-2}\left(\xi^{\infty}\right)$.

Thus we can construct an almsot Kählerian space $V^{2 n}$ with $F_{\dot{B}}{ }^{A}=\nabla_{B} u^{A}$ starting from a family of some almost Kählerian spaces $\stackrel{*}{V}^{2 n-2}\left(\xi^{\infty}\right)$. We can even construct an almost Kählerian space $V^{2 n}$ from only one almost Kählerian space $\stackrel{*}{V}^{2 n-2}$. We need only to consider that $g_{j i}$ do not involve $\xi^{\infty}$. There is an intrinsic difference between the two cases, for the variables $\xi^{0}, \xi^{1}, \ldots, \xi^{2 n-2}$ and the variable $\xi^{\infty}$ are completely separated in (2. 4).

Consequently we have the following theorems.

THEOREM 4.1. Let $V^{2 n}$ be a Riemannian space which becomes an almost Kählerian space by virtue of a tensor field $\nabla_{b} u^{A}$. Then we get a family of almost Kählerian spaces $\stackrel{*}{V}^{2 n-2}$. If we take in $V^{2 n}$ a favourable coordinate system $\left(\xi^{A}\right)$, then the members of the family are labelled by $\xi^{\infty}$ and $V^{* 2 n-2}\left(\xi^{\infty}\right)$ has the fundamental tensor

$$
g_{j i}=\frac{G_{j i}}{G_{00}}-\frac{G_{0 j} G_{0 i}}{\left(G_{00}\right)^{2}}
$$

and the almost complex structure (4.9). If we consider a hypersurface $\xi^{\infty}=$ const. in $V^{2 n}$ and regard in it each trajectory of the group of motions induced by the Killing vector $u^{A}$ as a point, we get a Riemannian space of

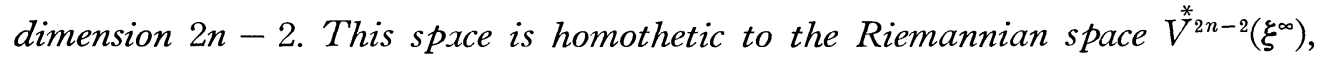
the ratio of the metric tensor being $\xi^{\infty}: 1$.

THEOREM 4.2. Let us consider a family of Riemannian spaces $\stackrel{*}{M}\left(\xi^{\infty}\right)$ of dimension $2 n-2$, where $\xi^{\infty}$ is a parameter indicating the space and $\xi^{1}, \ldots, \xi^{2 n-2}$ are coordinates of a point. The fundamental tensor of $M^{*}\left(\xi^{\infty}\right)$ is denoted by

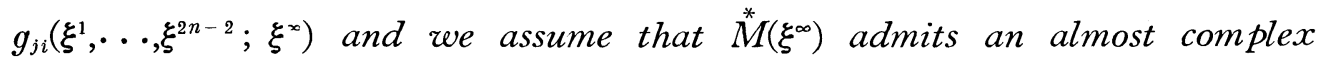
structure

$$
f_{j}^{i}=f_{j k} g^{k i}, \quad f_{j i}=\frac{1}{2}\left(\partial_{j} f_{i}-\partial_{i} f_{j}\right)
$$


derived from a covariant vector field $f_{i}$, where $f_{i}$ do not involve the variable $\xi^{\infty}$. Then, if $G_{B A}$ satisfies (4.10), a Riemannian space of dimension $2 n$ obtained by introducing the fundamental tensor $G_{B A}$ into the space $\left\{M^{*}\left(\xi^{\infty}\right) \times \xi^{0} \times \xi^{\infty} ; \xi^{0} \in D_{0}\right.$, $\left.\xi^{\infty} \in D_{1}\right\}$ admits a Killing vector $u^{A}=\delta_{0}^{A}$ and it becomes an almost Kählerian space by virtue of the tensor $\nabla_{B} u^{A}, M_{M}^{*}\left(\xi^{\infty}\right)$ playing the role of $V^{* 2 n-2}\left(\xi^{\infty}\right)$. We may consider a special case such that $g_{j i}$ do not involve $\xi^{\infty}$.

We remark that we have in favourable coordinates

$$
F_{j}^{i}=f_{j}^{i}, F_{\infty}^{i}=0, F_{\dot{0}}^{i}=0 .
$$

This is proved as follows.

$$
\begin{aligned}
F_{j^{i}} & =\nabla_{j} u^{i}=\left\{\begin{array}{c}
i \\
j 0
\end{array}\right\}=G^{i h}[j 0, h]=\frac{1}{2} G^{i h}\left(\partial_{j} G_{0 h}-\partial_{h} G_{0 j}\right) \\
& =\frac{1}{2} g^{i n}\left(\partial_{j} f_{h}-\partial_{h} f_{j}\right), \\
F_{\dot{\infty}}^{i} & =\left\{\begin{array}{c}
i \\
\infty 0
\end{array}\right\}=G^{i p}[\infty 0, p]=G^{i 0}[\infty 0,0]+G^{i h}[\infty 0, h] \\
& =\frac{1}{2} G^{i 0}+\frac{1}{2 \xi^{\infty}} G^{i h} G_{0 h}=0, \\
F_{\dot{0}}^{i} & =\left\{\begin{array}{c}
i \\
0
\end{array}\right\}=G^{i p}\left[\begin{array}{ll}
0 & 0, p]=0 .
\end{array}\right.
\end{aligned}
$$

(1. 3) and (4.11) also bring about the formula $f_{\dot{j}}{ }^{k} f_{\dot{k}}{ }^{i}=F_{\dot{j}}{ }^{k} F_{\dot{k}}{ }^{i}=F_{\dot{j}}{ }^{s}{F_{\dot{s}}}^{i}=-\delta_{j}{ }^{i}$.

5. A Riemannian space $V^{2 n}$ which admits a Killing vector $u^{A}$ satisfying $\left(\nabla_{B} u^{T}\right)\left(\nabla_{A} u_{T}\right)=G_{B A}$ and such that the hypersurfaces $u^{T} u_{T}=$ const. are totally umbilical. A necessary and sufficient condition that the hypersurfaces $\phi\left(x^{1}, \cdots, x^{m}\right)$ $=$ const. in a Riemannian space $V^{m}$ be totally umbilical is that $\varphi$ satisfy equations of the form

$$
\nabla_{j} \nabla_{i} \varphi=\alpha g_{j i}+\left(\nabla_{j} \varphi\right) B_{i}+\left(\nabla_{i} \varphi\right) B_{j} \quad i, j=1, \cdots, m .
$$

Hence, if the hypersurfaces $u^{T} u_{T}=$ const. in $V^{2 n}$ are totally umbilical, we have

$$
\nabla_{B} \nabla_{A}\left(u^{T} u_{T}\right)=\alpha G_{B A}+\left(\nabla_{B}\left(u^{T} u_{T}\right)\right) \Phi_{A}+\left(\nabla_{A}\left(u^{T} u_{T}\right)\right) \Phi_{B} .
$$

Since $u^{T} u_{T}=\xi^{\infty}$, the left hand side becomes

$$
\begin{aligned}
\partial_{B} \partial_{A} \xi^{\infty} & -\left\{\begin{array}{c}
C \\
B A
\end{array}\right\} \partial_{C} \xi^{\infty}=-\left\{\begin{array}{c}
\infty \\
B A
\end{array}\right\}=-G^{\infty \infty}[B A, \infty] \\
& =-2 \xi^{\infty}\left(\partial_{B} G_{A \infty}+\partial_{A} G_{B \infty}-\partial_{\infty} G_{B A}\right)
\end{aligned}
$$

and the right hand side becomes 


$$
\alpha G_{B A}+\delta_{B}^{\infty} \Phi_{A}+\delta_{A}^{\infty} \Phi_{B} .
$$

Hence a necessary and sufficient condition that $u^{T} u_{T}=$ const. be totally umbilical is that the following equations

$$
\partial_{\infty} G_{y x}=\frac{\alpha}{2 \xi^{\infty}} G_{y x}
$$

which are obtained by putting $B=y, A=x$, be satisfied. But, as we have (3. 8) already, we get $\alpha=2$, and (5.1) becomes

$$
\partial_{\infty} G_{y_{x}}=\left(\xi^{\infty}\right)^{-1} G_{y x} .
$$

Thus we obtain the following theorem.

THEOREM 5.1. A necessary and sufficient condition that in an almost Kählerian space $V^{2 n}$ with $F_{B}^{{ }^{A}}=\nabla_{B} u^{A}$ the hypersurfaces $u^{T} u_{T}=$ const. be totally umbilical is that $\left(\xi^{\infty}\right)^{-1} G_{j i}$ do not involve the variable $\xi^{\infty}$ in a favourable coordinate system.

We have proved also the next theorem.

THEOREM 5.2. Let us assume that a Riemannian space $V^{2 n}$ admits a Killing vector feld $\mathbf{u}$ such that $|\mathbf{u}|$ is not constant in $V^{2 n}$. Then a necessary and sufficient condition that the hypersurfaces $u^{T} u_{T}=$ const. be totally umbilical is that the equation $\partial_{\infty} G_{y_{x}}=\alpha G_{y_{x}}$ be satisfied for some function $\alpha$ in a favourable coordinate system.

Let us calculate some components of the curvature tensor by the formula

$$
\begin{aligned}
K_{D C B A} & =\frac{1}{2}\left(\partial_{D} \partial_{B} G_{C A}+\partial_{C} \partial_{A} G_{D B}-\partial_{D} \partial_{A} G_{C B}-\partial_{C} \partial_{B} G_{D A}\right) \\
& +G^{T S}\{[D B, T][C A, S]-[D A, T][C B, S]\} .
\end{aligned}
$$

Then, using (3. 2), (3. 3), (3. 5), we get

$$
\begin{aligned}
K_{0 B .40} & =G^{T S}\{[0 A, T][0 B, S]-[00, T][B A, S]\} \\
& =G_{B A}-G^{\infty \infty}[00, \infty][B A, \infty] \\
& =G_{B A}+\xi^{\infty}\left(\partial_{B} G_{A \infty}+\partial_{A} G_{B \infty}-\partial_{\infty} G_{B A}\right),
\end{aligned}
$$

hence

$$
K_{0 \infty \infty 0}=0, K_{0 x \infty 0}=0, K_{0 y x 0}=G_{y x}-\xi^{\infty} \partial_{\infty} G_{y x} .
$$

Consequently a necessary and sufficient condition that the hypersurfaces $\xi^{\infty}$ $=$ const. be totally umbilical can be written as $K_{0 y x_{0}}=0$. We can also obtain the same result directly if we use (1. 4), get $\nabla_{B} \nabla_{A}\left(u^{T} u_{T}\right)=2 G_{B A}-2 K_{T B A S} u^{T} u^{S}$ $=2 G_{B A}-2 K_{0 B A 0}$ and substitute the latter into $\nabla y \nabla x\left(u^{T} u_{T}\right)=\alpha G_{y_{x}}$.

Hence we get the

COROllary OF THEOREM 5.1. A necessary and sufficient condition 
that in an almost Kählerian space $V^{2 n}$ with $F_{\dot{B}}{ }^{A}=\nabla_{B} u^{A}$ the hypersurfaces $u^{T} u_{T}=$ const. be totally umbilical is that, among the components of the curvature tensor, $K_{0 B A 0}$ vanish in favourable coordinates.

6. A Kählerian space $V^{2 n}$ with the complex structure $F_{B}^{{ }^{A}}=\nabla_{B} u^{A}$. We calculate components $K_{0{ }^{C} B A}$ when $\left(\xi^{\infty}\right)^{-1} G_{y_{x}}$ do not contain the variable $\xi^{\infty}$.

We have already

$$
K_{0 B A 0}=0
$$

and we can prove

$$
K_{0 B A \infty}=0
$$

as follows.

If a Riemannian space of dimension $2 n$ admits a Killing vector $u^{A}$ satisfying (1. 1$)$, we get

$$
\frac{1}{2} \nabla \sigma \nabla_{B} \nabla_{A}\left(u^{T} u_{T}\right)=\nabla_{\sigma}\left(G_{B A}-K_{T_{B A S}} u^{T} u^{S}\right)=-\nabla_{\sigma}\left(K_{T_{B A S}} u^{T} u^{S}\right) .
$$

If (6. 1) holds in addition, we get $\nabla_{c} \nabla_{B} \nabla_{A}\left(u^{T} u_{T}\right)=0$ because of $K_{T B . A S} u^{T} u^{S}=0$. Hence we obtain $K_{\dot{\sigma} \dot{B} \dot{A}} \nabla_{S}\left(u^{T} u_{T}\right)=0$. Since $\nabla_{S}\left(u^{T} u_{T}\right)=\delta_{S}^{\infty}$, this equation can be written as follows,

$$
K_{\dot{C} \dot{B} \dot{A}}^{\infty}=K_{C B A \infty}=0,
$$

which contains (6. 2).

Thus, in order to get expressions of $K_{0 C B A}$, we only need to calculate $K_{0 j i h}$. of

Using equations such as (3. 2), (3. 8), (3.10) and (5. 2), the right hand side

$$
K_{0 j i h}=\frac{1}{2}\left(\partial_{j} \partial_{h} G_{0 i}-\partial_{j} \partial_{i} G_{0 h}\right)+G^{T S}\{[0 i, T][j h, S]-[0 h, T][j i, S]\}
$$

becomes

$$
\begin{aligned}
& \frac{1}{2} \partial_{j}\left(\partial_{h} G_{0 i}-\partial_{i} G_{0 h}\right)+\left(\xi^{\infty}\right)^{-1}\left(G_{0 i} G_{j h}-G_{0 h} G_{j i}\right) \\
& +\frac{1}{4} G^{k 0}\left[\left(\partial_{i} G_{0 k}-\partial_{k} G_{0 i}\right)\left(\partial_{j} G_{0 h}+\partial_{h} G_{0 j}\right)\right. \\
& \left.\quad-\left(\partial_{h} G_{0 k}-\partial_{k} G_{0 h}\right)\left(\partial_{j} G_{0 i}+\partial_{i} G_{0 j}\right)\right] \\
& +\frac{1}{4} G^{l k}\left[\left(\partial_{i} G_{0 l}-\partial_{l} G_{0 i}\right)\left(\partial_{j} G_{h k}+\partial_{h} G_{j k}-\partial_{k} G_{j h}\right)\right. \\
& \left.\quad-\left(\partial_{h} G_{0 l}-\partial_{l} G_{0 h}\right)\left(\partial_{j} G_{i k}+\partial_{i} G_{j k}-\partial_{k} G_{j i}\right)\right] .
\end{aligned}
$$

On the other hand, from (3. 9), (4.2) and (4. 3) we have 


$$
G^{l k}=\left(\xi^{\infty}\right)^{-1} g^{l k}, G_{j i}=\xi^{\infty}\left(g_{j i}+f_{j} f_{i}\right), G_{0 i}=\xi^{\infty} f_{i}
$$

where $f_{i}, g_{j i}, g^{l k}$ do not contain $\xi^{\infty}$. Hence, denoting the Christoffel symbols of the first kind formed from $g_{j i}$ by $[j i, h]^{*}$ we get

$$
\begin{gathered}
\partial_{j} G_{h k}+\partial_{h} G_{j k}-\partial_{k} G_{j h}=\xi^{\infty}\left\{2[j h, k]^{*}+f_{k}\left(\partial_{j} f_{h}+\partial_{h} f_{j}\right)\right. \\
\left.+f_{h}\left(\partial_{j} f_{k}-\partial_{k} f_{j}\right)+f_{j}\left(\partial_{h} f_{k}-\partial_{k} f_{h}\right)\right\} .
\end{gathered}
$$

We substitute these equations into the formula obtained above, take (4.4) into account, and get

$$
\begin{aligned}
K_{0 j i h}= & \frac{1}{2} \xi^{\infty} \partial_{j}\left(\partial_{h} f_{i}-\partial_{i} f_{h}\right)+\xi^{\infty}\left(f_{i} g_{j h}-f_{h} g_{j i}\right) \\
+ & \frac{1}{4} \xi^{\infty}\left(\xi^{\infty} G^{0 k}+f_{l} g^{l k}\right)\left[\left(\partial_{i} f_{k}-\partial_{k} f_{i}\right)\left(\partial_{j} f_{h}+\partial_{h} f_{j}\right)\right. \\
& \left.\quad-\left(\partial_{h} f_{k}-\partial_{k} f_{h}\right)\left(\partial_{j} f_{i}+\partial_{i} f_{j}\right)\right] \\
+ & \frac{1}{2} \xi^{\infty}\left(\partial_{i} f_{k}-\partial_{k} f_{i}\right)\left\{\begin{array}{c}
k \\
j h
\end{array}\right\}^{*}-\frac{1}{2} \xi^{\infty}\left(\partial_{h} f_{k}-\partial_{k} f_{h}\right)\left\{\begin{array}{c}
k \\
j i
\end{array}\right\}^{*} \\
+ & \xi^{\infty}\left(f_{h} g_{j i}+f_{j} g_{h i}-f_{i} g_{j h}-f_{j} g_{i h}\right) .
\end{aligned}
$$

Since we have $\xi^{\infty} G^{0 k}+f_{l} g^{l k}=0$ from (4. 3), we easily get

$$
K_{0 j i h}=-\xi^{\infty} \stackrel{*}{\nabla} f_{i h} .
$$

Hence we obtain the

THEOREM 6.1. If in an almost Kählerian space $V^{2 n}$ with $F_{B}^{A 4}=\nabla_{B} u^{A}$ the hypersurfaces $u^{T} u_{T}=$ const. are totally umbilical, the curvature tensor satisfy (6. 1), (6. 2) and (6.6) in favourable coordinates.

A necessary condition that the hypersurfaces $u^{T} u_{T}=$ const. be totally umbilical in such $V^{2 n}$ is that (5.2) hold. But we can replace this by $K_{0 B A 0}=0$ by virtue of ( 5 .3). Hence we obtain the

COROLLARY. A necessary and sufficient condition that in an almost Kählerian space $V^{2 n}$ with $F_{\dot{B}}{ }^{A}=\nabla_{B} u^{A}$ the hypersurfaces $u^{T} u_{T}=$ const. be totally umbilical is that among the components $K_{D C B A}$ of the curvature tensor, $K_{0 j i n}$ satisfy (6. 6) and other components of the form $K_{0 C B A}$ all vanish in favourable coordinates.

If $\stackrel{*}{\nabla} f_{i h}=0$, then $\stackrel{*}{V^{2 n-2}}$ is a Kählerian space. Hence we get the

THEOREM 6.2. A necessary and sufficient condition that an almost Kählerian space $V^{2 n}$ with ${F_{B}}^{A}=\nabla_{B} u^{A}$ be a Kählerian space is that the spaces 
$\stackrel{*}{V}^{2 n-2}$ be Kählerian spaces beside that the hypersurfaces $u^{T} u_{T}=$ const. be totally umbilical.

PROOF. That the space $V^{2 n}$ be Kählerian is equivalent to that the tensor $\nabla_{C} \Gamma_{B} u^{A}$ vanish, that is, $K_{\dot{S} \dot{C} \dot{B}}{ }^{A} u^{S}=0$, or $K_{0 C B A}=0$. Hence, if $V^{2 n}$ is Kählerian, we get (5. 2) from (5. 3) and the hypersurfaces $u^{T} u_{T}=$ const. are totally umbilical. $V^{2 n-2}$ are Kählerian by virtue of $(6.6)$. The converse is easily proved by using (6. 1), (6. 2) and (6. 6).

7. Holomorphic sectional curvature in the direction orthogonal to $\nabla^{A}\left(u^{T} u_{T}\right)$ and $u^{A}$. At first we prove the

LEMMA 7.1. Let $V^{2 n}$ be an almost Kählerian space such that $F_{\dot{B}}{ }^{A}=\nabla_{B} u^{A}$. Then, if a direction $v^{A}$ is orthogonal to $u^{A}$ and $\nabla^{A}\left(u^{T} u_{T}\right), v^{B} F_{\dot{B}}{ }^{A}$ is also orthogonal to $u^{A}, \nabla^{A}\left(u^{T} u_{T}\right)$.

Proof is easily obtained from $v^{B} F_{B}^{A} u_{A}=v^{B}\left(\nabla_{B} u^{A}\right) u_{A}=\frac{1}{2} v^{B} \nabla^{B}\left(u^{T} u_{T}\right)$ $=0, v^{B} F_{\dot{B}}^{A} \nabla_{A}\left(u^{T} u_{T}\right)=2 v^{B} F_{\dot{B}}^{A} F_{\dot{A}} u_{T}=-2 v^{B} u_{B}=0$.

In a Kählerian space $V^{2 n}$ with $F_{\dot{B}}{ }^{A}=\nabla_{B} u^{A}$ we have obtained $K_{S C B A} u^{S}$ $=K_{0 C_{B A}}=0$ in favourable coordinates. Consequently we also obtain (6. 3) from $\nabla_{C} \nabla_{B} \nabla_{A}\left(u^{T} u_{T}\right)=0$ and $K_{T B A S} u^{T} u^{S}=0$. Thus we get the

THEOREM 7.1. Let $V^{2 n}$ be a Kählerian space with $F_{\dot{B}}{ }^{A}=\nabla_{B} u^{A}$. Then, if we take a favourable coordinate system, the components $K_{D C B A}$ of the curvature tensor vanish except those of the form $K_{k j i n}$.

Let us study the holomorphic sectional curvature $K(v)$ with respect to a direction $v^{A}$ which is orthogonal to both $u^{A}$ and $\nabla^{A}\left(u^{T} u_{T}\right)$. According to Theorem 7.1 we get at first

$$
K(v)=-\frac{K_{k j i h} F_{\dot{T}}{ }^{k} F_{\dot{S}}{ }^{i} v^{T} v^{S} v^{j} v^{h}}{G_{D C} v^{D} v^{C} G_{B . A} v^{B} v^{A}} .
$$

On the other hand we have (4.11), hence we get

$$
K(v)=-\frac{K_{k j i n} f_{\dot{m}}{ }^{k} f_{i}{ }^{i} v^{m} v^{l} v^{j} v^{h}}{G_{D C} v^{D} v^{C} G_{B A} v^{B} v^{A}} .
$$

Since the vector $v^{A}$ is orthogonal to vectors $u^{A}$ and $\nabla^{A}\left(u^{T} u_{T}\right)$, we have $v_{0}=0, v^{\infty}=0$. From $v_{0}=G_{00} v^{0}+G_{0 l} v^{l}=0$ we get

$$
v^{0}=-\left(\xi^{\infty}\right)^{-1} G_{0 l} v^{l}=-f_{l} v^{l}
$$

a nd

$$
\begin{aligned}
& G_{B A} v^{B} v^{A}=G_{00} v^{0} v^{0}+2 G_{0 l} v^{0} v^{l}+G_{m l} v^{m} v^{l} \\
& \quad=\left\{G_{m l}-\left(\xi^{\infty}\right)^{-1} G_{0 m} G_{0 l}\right\} v^{m} v^{l}
\end{aligned}
$$


Consequently we have

$$
=\xi^{\infty} g_{m l} v^{m} v^{l} \text {. }
$$

$$
K(v)=-\frac{K_{k j i h} f_{\dot{m}}^{k} f_{i}^{i} v^{m} v^{l} v^{j} v^{h}}{\left(\xi^{\infty}\right)^{2} g_{k j} v^{k} v^{j} g_{i h} v^{i} v^{h}}
$$

and the

THEOREM 7.2. Let $V^{2 n}$ be a Kählerian space with $F_{\dot{B}}{ }^{A}=\nabla_{B} u^{A}$. Then, for any vector $v^{A}$ orthogonal to vectors $u^{A}$ and $\nabla^{A}\left(u^{T} u_{T}\right)$, the holomorphic sectional curvature $K(v)$ satisfies (7. 2) in a favourable coordinate system.

Let us study a necessary and sufficient condition that $K(v)$ do not depend upon the direction $v^{A}$ as long as $v^{A}$ is orthogonal to $u^{A}$ and $\nabla^{A}\left(u^{T} u_{T}\right)$. The way of deduction is similar to the one of Yano [4],p.239.

At first we have

$$
K_{k j i h} F_{\dot{m}}^{k} F_{i}{ }^{i} v^{m} v^{l} v^{j} v^{h}=-k^{\prime} g_{m j} v^{m} v^{j} g_{l h} v^{l} v^{h},
$$

where $k^{\prime}$ is independent of $v^{i}$, for we have $v^{\infty}=0$ and $v^{0}$ is determined by (7. 1), while $v^{i}$ are arbitrary.

Since $V^{2 n}$ is a Kählerian space, we have

$$
\begin{gathered}
K_{\dot{D} \dot{C} \dot{B}}{ }^{S} F_{\dot{S}}{ }^{A}=K_{\dot{D} \dot{C} \dot{S}}{ }^{A} F_{\dot{B}}{ }^{S}, K_{D C B S} F_{\dot{A}}{ }^{S}=K_{D C A S} F_{\dot{B}}{ }^{S}, \\
K_{D C B A}=K_{D C T_{S}} F_{\dot{B}}{ }^{T} F_{\dot{A}}{ }^{S},
\end{gathered}
$$

from which we get, using (4.11),

$$
K_{\dot{k} j i}{ }^{l} F_{i}{ }^{h}=K_{\dot{k} j i^{h}} F_{i}{ }^{l}, K_{k j i l} F_{\dot{b}^{l}}{ }^{l}=K_{k j h l} F_{i}{ }^{l}
$$

and

$$
K_{k j i h}=K_{k j m l} F_{i}{ }^{m} F_{h^{l}}{ }^{l} .
$$

We also have the identity $K_{k j i h}=K_{i n k j}$.

From (7. 3) we find that $F_{m j l h}=K_{k j i h} F_{\dot{m}}{ }^{k} F_{i}{ }^{i}$ is symmetric in $m$ and $j$ and also in $l$ and $h$. Since we have moreover $F_{m j l h}=F_{l h m j}$, we get

$$
\begin{array}{r}
K_{k j i h} F_{\dot{m}}{ }^{k} F_{i}{ }^{i}+K_{k l i j} F_{\dot{m}}{ }^{k}{F_{\dot{h}}}^{i}+K_{k h i l} F_{\dot{m}}{ }^{k} F_{\dot{j}}{ }^{i} \\
=-k^{\prime}\left(g_{m j} g_{l h}+g_{m l} g_{h j}+g_{m h} g_{j l}\right) .
\end{array}
$$

On the other hand we have $F_{j}{ }^{l} g_{l i}=f_{j}{ }^{l} g_{l i}=f_{j i}$. Hence we get

$$
\begin{aligned}
K_{k j i h} & -K_{k m l j} F_{i}^{m} F_{\dot{h}^{l}}-K_{k h m l} F_{\dot{j}}{ }^{m} F_{i^{l}}{ }^{2} \\
& =-k^{\prime}\left(f_{k j} f_{i h}+g_{k i} g_{j h}+f_{k h} f_{i j}\right) .
\end{aligned}
$$

Subtracting from this equation an equation obtained by interchanging $i$ and $h$, we get

$$
2 K_{k j i h}+K_{k j m l} F_{i}{ }^{m} F_{\dot{h}}{ }^{l}-K_{k h m l} F_{j}{ }^{m} F_{i}^{l}+K_{k i m l} F_{j}{ }^{m} F_{\dot{h}^{l}}
$$




$$
=-k^{\prime}\left(2 f_{k j} f_{i h}+f_{k i} f_{j h}-f_{k h} f_{j i}+g_{k i} g_{j h}-g_{k h} g_{j i}\right) \text {. }
$$

The left hand side being equal to $4 K_{k j i h}$ on account of (7. 4), we obtain

$$
\left.K_{k j i h}=\frac{k^{\prime}}{4}\left(g_{k h} g_{j i}-g_{j h} g_{k i}\right)+\left(f_{k h} f_{j i}-f_{j h} f_{k i}\right)-2 f_{k j} f_{i h}\right]
$$

as a necessary condition that $K(v)$ do not depend upon $v^{i}$. This is evidently a sufficient condition as long as we consider the concition at each point of $V^{2 n}$.

Now, we shall consider $k^{\prime}$ in (7.5) as a function of the point and prove the

THEOREM 7.3. Let us consider a Kählerian space $V^{2 n}$ with $F_{\dot{B}}{ }^{A}=\nabla_{B} u^{A}$ and take a favourable coordinate system. A necessary and sufficient condition that the holomorphic sectional curvature with respect to a variable direction which is orthogonal to both $u^{A}$ and $\nabla^{A}\left(u^{T} u_{T}\right)$ be constant at each point is that the curvature tensor $K_{D C B A}$ satisfy the following equations in which $k$ is a constant.

$$
\left.K_{k j i h}=\frac{1}{4} k \xi^{\infty}\left(g_{k h} g_{j i}-g_{j h} g_{k i}\right)+\left(f_{k h} f_{j i}-f_{j h} f_{k i}\right)-2 f_{k j} f_{i h}\right] .
$$

For this purpose we only need to show that we can deduce (7. 6) from (7. 5). We write Bianchi's identity in the form

$$
\begin{aligned}
& \partial_{E} K_{D C B A}+\partial_{D} K_{C E B A}+\partial_{C} K_{F D B A} \\
& -\left\{\begin{array}{c}
S \\
E B
\end{array}\right\} K_{D C S A}-\left\{\begin{array}{c}
S \\
D B
\end{array}\right\} K_{C E S A}-\left\{\begin{array}{c}
S \\
C B
\end{array}\right\} K_{E D S A} \\
& -\left\{\begin{array}{c}
S \\
E A
\end{array}\right\} K_{D C B S}-\left\{\begin{array}{c}
S \\
D A
\end{array}\right\} K_{C E B S}-\left\{\begin{array}{c}
S \\
C A
\end{array}\right\} K_{E D B S}=0 .
\end{aligned}
$$

Noticing that the components $K_{D^{\prime} \boldsymbol{B} B \boldsymbol{A}}$ vanish except $K_{k j i h}$ and putting $E=\infty$, $D=k, C=j, B=i, A=h$, we get

$$
\partial_{\infty} K_{k j i h}-\left\{\begin{array}{c}
l \\
\infty i
\end{array}\right\} K_{k j l h}-\left\{\begin{array}{c}
l \\
\infty h
\end{array}\right\} K_{k j i l}=0 .
$$

Since we have

$$
\begin{aligned}
\left\{\begin{array}{c}
l \\
\infty i
\end{array}\right\} & =G^{l 0}[\infty i, 0]+G^{l k}[\infty i, k] \\
& =\frac{1}{2 \xi^{\infty}}\left(G^{l 0} G_{i 0}+G^{l k} G_{i k}\right)=\frac{1}{2 \xi^{\infty}} \delta_{i}^{l},
\end{aligned}
$$

we get $\partial_{\infty} K_{k j i h}=\left(\xi^{\infty}\right)^{-1} K_{k j i h}$, which proves that $k=\left(\xi^{\infty}\right)^{-1} k^{\prime}$ does not involve $\xi^{\infty}$. 
We now prove that $k$ does not involve $\xi^{i}$.

By straightforward calculation using (6.4) and (6.5) we obtain

$$
\begin{aligned}
& \partial_{l} K_{k j i h}-\left\{\begin{array}{l}
m \\
l i
\end{array}\right\} K_{k j m h}-\left\{\begin{array}{c}
m \\
l
\end{array}\right\} K_{k j i m} \\
& =\partial_{l} K_{k j i h}-\left(G^{0 m}[l i, 0]+G^{n m}[l i, n]\right) K_{k j m h} \\
& -\left(G^{0 m}[l h, 0]+G^{n m}[l h, n]\right) K_{k j i m} \\
& =\partial_{l} K_{k j i h}-\left\{\begin{array}{l}
m \\
l i
\end{array}\right\}^{*} K_{k j m h}-\left\{\begin{array}{l}
m \\
l h
\end{array}\right\}^{*} K_{k j i m} \\
& -\left[-f^{m} \frac{1}{2}\left(\partial_{i} f_{l}+\partial_{l} f_{i}\right)+g^{n m} \frac{1}{2}\left\{f_{n}\left(\partial_{l} f_{i}+\partial_{i} f_{l}\right)\right.\right. \\
& \left.\left.+f_{i}\left(\partial_{l} f_{n}-\partial_{n} f_{l}\right)+f_{l}\left(\partial_{i} f_{n}-\partial_{n} f_{i}\right)\right\}\right] K_{k j m h} \\
& -\left[-f^{m} \frac{1}{2}\left(\partial_{h} f_{l}+\partial_{l} f_{h}\right)+g^{n m} \frac{1}{2}\left\{f_{n}\left(\partial_{l} f_{h}+\partial_{h} f_{l}\right)\right.\right. \\
& \left.\left.+f_{h}\left(\partial_{l} f_{n}-\partial_{n} f_{l}\right)+f_{l}\left(\partial_{h} f_{n}-\partial_{n} f_{h}\right)\right\}\right] K_{k j i m}
\end{aligned}
$$

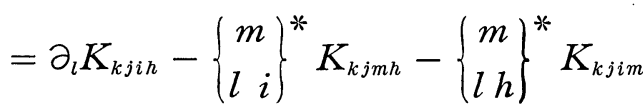

$$
\begin{aligned}
& -\frac{g^{n m}}{2}\left\{f_{i}\left(\partial_{l} f_{n}-\partial_{n} f_{l}\right)+f_{l}\left(\partial_{i} f_{n}-\partial_{n} f_{i}\right)\right\} K_{k j m h} \\
& -\frac{g^{n m}}{2}\left\{f_{h}\left(\partial_{l} f_{n}-\partial_{n} f_{l}\right)+f_{l}\left(\partial_{h} f_{n}-\partial_{n} f_{h}\right)\right\} K_{k j i m} \\
& =\partial_{l} K_{k j i h}-\left\{\begin{array}{l}
m \\
l i
\end{array}\right\}^{*} K_{k j m h}-\left\{\begin{array}{l}
m \\
l h
\end{array}\right\}^{*} K_{k j i m} \\
& -\left(f_{i} f_{i}{ }^{m}+f_{l} f_{\dot{i}}{ }^{m}\right) K_{k j m h}-\left(f_{h} f_{i}{ }^{m}+f_{l} f_{\dot{h}^{m}}{ }^{m}\right) K_{k j i m} .
\end{aligned}
$$

Hence we obtain the following equation when we put $E=l, D=k, C=j$, $B=i, A=h$ in (7. 7),

$$
\begin{aligned}
& \stackrel{*}{\nabla}^{*} K_{k j i h}+\stackrel{*}{\nabla}_{k} K_{j l i h}+\stackrel{*}{\nabla}_{j} K_{l k i h} \\
& \quad-K_{k j m h}\left(f_{i}^{m} f_{i}+f_{i}^{m} f_{l}\right)+K_{k j m i}\left(f_{i^{m}} f_{h}+f_{\dot{h}^{m}} f_{l}\right) \\
& \quad-K_{j l m h}\left(f_{\dot{k}}^{m} f_{i}+f_{i}^{m} f_{k}\right)+K_{j l m i}\left(f_{\dot{k}}^{m} f_{h}+f_{\dot{i}}^{m} f_{k}\right) \\
& \quad-K_{l k m h}\left(f_{\dot{j}}^{m} f_{i}+f_{\dot{i}^{m}}^{m} f_{j}\right)+K_{l k m i}\left(f_{\dot{j}}^{m} f_{h}+f_{\dot{b}}^{m} f_{j}\right)=0 .
\end{aligned}
$$

In this equation $\stackrel{*}{\nabla}_{l} K_{k j i h}$ denotes a covariant derivative obtained formally by

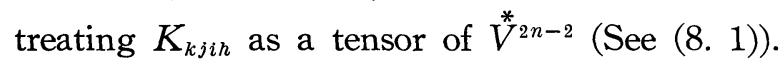

But we have from (7. 3) 


$$
K_{k j m h} f_{i}^{m}-K_{k j m i} f_{\dot{h}}^{m}=0
$$

and

$$
\begin{aligned}
& K_{k j m h} f_{i^{m}}+K_{j l m h} f_{\dot{k}}{ }^{m}+K_{l k m h} f_{\dot{j}}{ }^{m} \\
& \quad=\left(K_{k j m l}+K_{j l n n k}+K_{l k m j}\right) f_{\dot{h}}{ }^{m} \\
& \quad=-3 K_{[k j l] m} f_{\dot{h}^{m}}{ }^{m}=0,
\end{aligned}
$$

hence we get

$$
\stackrel{*}{\nabla}_{l} K_{k j i h}+\stackrel{*}{\nabla}_{k} K_{j l i h}+\stackrel{*}{\nabla}_{j} K_{l k i h}=0 .
$$

This equation is formally the same with Bianchi's identity in $\stackrel{*}{V}^{2 n-2}$. Hence, substituting (7. 6) into this equation, we find that $k \xi^{\infty}$ does not contain the variables $\xi^{1}, \cdots, \xi^{2 n-2}$. Thus we obtain $k=$ const. and Theorem 7.3 is proved.

We have also proved the following theorem.

THEOREM 7.4. We consider a Kählerian space $V^{2 n}$ with $F_{\dot{B}}{ }^{A}=\nabla_{B} u^{A}$ and take a favourable coordinate system. Then the functions $\left(\xi^{\infty}\right)^{-1} K_{k j i h}$ do not involve the variable $\xi^{\infty}$ and $K_{k j i n}$ satisfy (7. 8) if $\stackrel{*}{\nabla}_{l}$ denotes formal covariant differentiation with respect to the Christoffel symbols formed from $g_{j i}$.

8. A relation between the curvature of $\stackrel{*}{V}^{2 n-2}$ and the curvature of $V^{2 n}$. We can prove by straightforward calculation the following theorem.

THEOREM 8.1. Let $V^{2 n}$ be an almost Kählerian space such that $F_{\dot{B}}{ }^{A}$ $=\nabla_{B} u^{A}$. If $\left(\xi^{\infty}\right)^{-1} G_{j i}$ do not depend on $\xi^{\infty}$ in favourable coordinates, then we have the following relation between the curvature tensor $\stackrel{*}{K}_{k j i n}$ of $\stackrel{*}{V}^{2 n-2}$ and the curvature tensor $K_{D C B A}$ of $V^{2 n}$,

$$
\begin{aligned}
\left(\xi^{\infty}\right)^{-1} & K_{k j i h}-\stackrel{*}{K}_{k j i h} \\
= & -g_{k h} g_{j i}+g_{j h} g_{k i}-\left(f_{k h} f_{j i}-f_{j h} f_{k i}\right)+2 f_{k j} f_{i h} \\
& +f_{j}^{*} \stackrel{*}{\nabla}_{k} f_{i h}-f_{k} \stackrel{*}{\nabla}_{j} f_{i h}+f_{h} \stackrel{*}{\nabla}_{i} f_{k j}-f_{i} \vec{\nabla}_{h} f_{k j} .
\end{aligned}
$$

From this theorem and Theorem 7.3 we get the

THEOREM 8.2. Let $V^{2 n}$ be a Kählerian space with $F_{\dot{B}}{ }^{A}=\nabla_{B} u^{A}$. A necessary and sufficient condition that the holomorphic sectional curvature $K(v)$ with respect to a direction $v^{A}$ orthogonal to $u^{A}$ and $\nabla^{A}\left(u^{T} u_{T}\right)$, when considered at each point, be independent of $v^{4}$ is that each Kählerian space $\stackrel{*}{V}^{2 n-2}$ be a space of constant holomorphic sectional curvature.

PROOF OF THEOREM 8.1. We start from

$$
K_{k j i h}=\frac{1}{2}\left[\partial_{k} \partial_{i} G_{j h}+\partial_{j} \partial_{h} G_{k i}-\partial_{k} \partial_{h} G_{j i}-\partial_{j} \partial_{i} G_{k h}\right]
$$




$$
\begin{aligned}
& +G^{\infty \infty}\{[k i, \infty][j h, \infty]-[k h, \infty][j i, \infty]\} \\
& +G^{00}\{[k i, 0][j h, 0]-[k h, 0][j i, 0]\} \\
& +G^{0 l}\{[k i, 0][j h, l]-[k h, 0][j i, l]\} \\
& +G^{0 l}\{[k i, l][j h, 0]-[k h, l][j i, 0]\} \\
& +G^{m l}\{[k i, m][j h, l]-[k h, m][j i, l]\}
\end{aligned}
$$

and as in $\$ 6$ take into account that $g_{j h}$ and $f_{j}$ do not contain $\xi^{\infty}$. Using relations such as (6. 4), (6. 5) again, we get after calculation

$$
\begin{aligned}
& \left(\xi^{\infty}\right)^{-1} K_{k j i h}-\stackrel{*}{K}_{k j i h} \\
& =2 f_{k j} f_{i h}+f_{j} \partial_{k} f_{i h}-f_{k} \partial_{j} f_{i h}+f_{h} \partial_{i} f_{k j}-f_{i} \partial_{h} f_{k j}+f_{k h} f_{i j}-f_{k i} f_{h j} \\
& +g_{k i} g_{j h}-g_{k h} g_{j i}+f_{k} f_{i} g_{j h}+f_{j} f_{h} g_{k i}-f_{k} f_{h} g_{j i}-f_{j} f_{i} g_{k h} \\
& +g^{m l}\left[\left(f_{k m} f_{i}+f_{i m} f_{k}\right)\left(f_{j l} f_{h}+f_{h l} f_{j}\right)\right. \\
& \left.-\left(f_{k m} f_{h}+f_{h m} f_{k}\right)\left(f_{j l} f_{i}+f_{i l} f_{j}\right)\right] \\
& +\left[-\left\{\begin{array}{c}
l \\
k i
\end{array}\right\}^{*} f_{l j}-\left\{\begin{array}{c}
l \\
j i
\end{array}\right\}^{*} f_{k l}\right] f_{h}+\left[-\left\{\begin{array}{c}
l \\
k i
\end{array}\right\}^{*} f_{l h}-\left\{\begin{array}{c}
l \\
k h
\end{array}\right\}^{*} f_{i l}\right] f_{j} \\
& -\left[-\left\{\begin{array}{c}
l \\
k h
\end{array}\right\}^{*} f_{l j}-\left\{\begin{array}{c}
l \\
j h
\end{array}\right\}^{*} f_{k l}\right] f_{i}-\left[-\left\{\begin{array}{c}
l \\
j i
\end{array}\right\} f^{*} f_{l h}-\left\{\begin{array}{c}
l \\
j h
\end{array}\right\}^{*} f_{i l}\right] f_{k}
\end{aligned}
$$

where

$$
\begin{aligned}
\stackrel{*}{K}_{k j i h}= & \frac{1}{2}\left[\partial_{k} \partial_{i} g_{j h}+\partial_{j} \partial_{h} g_{k i}-\partial_{k} \partial_{h} g_{j i}-\partial_{j} \partial_{i} g_{k h}\right] \\
& +g^{m l}\left\{[k i, m]^{*}[j h, l]^{*}-[k h, m]^{*}[j i, l]^{*}\right\}
\end{aligned}
$$

are the components of the curvature tensor of $V^{* 2 n-2}$. Since we have moreover $g^{m l} f_{k m} f_{j l}=g_{k j}$ and $\stackrel{*}{\nabla}_{k} f_{i h}=\partial_{k} f_{i h}-\left\{\begin{array}{c}l \\ k i\end{array}\right\} f_{l h}-\left\{\begin{array}{c}l \\ k h\end{array}\right\} f^{*}$, we obtain (8. 1).

(8. 1) also proves Theorem 7.4 directly.

\section{REFERENCES}

[1] L. P. EISENHART, Continuous groups of transformations, Princeton University Press, 1933.

[2] S. TACHIBANA, On almost-analytic vectors in almost-Kählerian manifolds, Tôhoku Math. Journ., 11(1959), 247-265.

[3] K. YANO, Groups of transformations in generalized spaces, Akademeia Press, Tokyo, 1949.

[4] K. YANO, The theory of Lie derivatives and its applications, North-Holland Publishing Co., Amsterdam, 1957.

YOKOHAMA NATIONAI. UNIVERSITY. 